\section{European Psychiatry}

www.cambridge.org/epa

\section{Viewpoint}

Cite this article: Bianchi R, Schonfeld IS (2021). Who needs to be "burned-out"? Time for a new approach to job-related distress. European Psychiatry, 64(1), e65, 1-2 https://doi.org/10.1192/j.eurpsy.2021.2240

Received: 15 September 2021

Accepted: 27 September 2021

\section{*Author for correspondence:}

Renzo Bianchi,

E-mail: renzo.bianchi@unine.ch

\title{
Who needs to be "burned-out"? Time for a new approach to job-related distress
}

Renzo Bianchi ${ }^{1 \star}$ (1) and Irvin Sam Schonfeld ${ }^{2}$ (B)

${ }^{1}$ Institute of Work and Organizational Psychology, University of Neuchâtel, Neuchâtel, Switzerland and ${ }^{2}$ Department of Psychology, The City College of the City University of New York, New York, New York, USA

Burnout is a popular indicator of job-related distress, notably in research on the ill-being of medical professionals $[1,2]$. The burnout construct is, however, plagued by definitional and measurement problems [1-3]. Often unnoticed, these problems undermine findings and conclusions emanating from burnout research [2,3]. This state of affairs is of concern. The definitional and measurement problems affecting the burnout construct hamper knowledge growth and waste resources $[1,2]$. These problems eventually impede our ability to make informed decisions and take effective action to support personnel [2,3]. In this paper, we argue that it is time for occupational health specialists to abandon the idea of burnout and focus on occupational depression.

\section{Drowned in Confusion}

Despite nearly 50 years of research, burnout remains loosely characterized and undiagnosable [1-3]. Consequently, it is impossible to identify individuals with burnout or estimate burnout's prevalence validly and reliably [1-3]. People do not always realize that published estimates of burnout's prevalence are based on clinically arbitrary, theoretically groundless, and highly heterogeneous identification criteria [3]. A recent review dedicated to physician burnout found no fewer than 142 unique categorizations of burnout, with prevalence estimates varying from 0 to $90 \%$ [1]. The diagnostic chaos surrounding burnout is equally problematic when it comes to developing individual treatments and organizational interventions [2].

Confusion does not dissipate when turning to the dominant, "dimensional" view of burnout reflected in the Maslach Burnout Inventory (MBI) [4]. Within the MBI framework, burnout is defined as a syndrome combining exhaustion, cynicism, and inefficacy. In contradiction with their own definition, however, the developers of the MBI have expressly recommended that exhaustion, cynicism, and inefficacy indices be analyzed and interpreted separately. It is important to spell out the implications of this inconsistency. When using the MBI based on its manual's guidelines, investigators end up with three entities (exhaustion, cynicism, and inefficacy), of which none is burnout. Put differently, when used in compliance with its developers' recommendations, the MBI is not a measure of burnout.

Many critics of the MBI have argued that exhaustion is, in fact, the only defining feature of burnout. From this perspective, cynicism and inefficacy constitute possible consequences of exhaustion rather than central characteristics of burnout. Consistent with this observation, a recent study involving a consortium of health experts from 29 countries found that burnout is generally equated with exhaustion [5]. Problematically, there is robust evidence that exhaustion reflects a classical depressive response to intractable (job) stress, not a new or unique phenomenon [3]. These findings question burnout's construct validity at its core.

One might claim that burnout is a euphemism for depression that, at least, allows individuals to speak more safely about job-related distress. Available evidence does not support such a view. The burnout label constitutes a barrier to employment and promotion, suggesting considerable stigma [6]. Even in terms of social representations, it is thus difficult to identify a clear advantage to using the burnout construct.

\section{Beyond Burnout}

Continuing to rely on the burnout construct holds little promise. The problems affecting the entity are so profound that they are unlikely to be resolvable, as suggested by nearly five decades of underwhelming progress in burnout research [7]. Most studies of burnout use the construct uncritically, as if its flaws and shortcomings were of negligible importance.

To assess and manage job-related distress more effectively, we advocate for a change in paradigm from burnout to occupational depression. A measure of job-ascribed depressive symptoms is now available, which may facilitate such a change [8,9]. Called the Occupational Depression Inventory (ODI), the instrument examines each core symptom of major depression through items incorporating attributions to work. The tool notably assesses work-related suicidal thoughts-a sign of severe job-related distress. Burnout measures ignore such crucial 
symptoms [3]. The ODI exhibits strong psychometric and structural properties and is available to specialists at no cost [8]. Instead of treating dimensional and categorical views of psychopathology as antinomic, the ODI incorporates both perspectives. The instrument allows for continuum-based and diagnostic approaches to job-related distress $[8,10]$.

We emphasize that a paradigm shift from burnout to occupational depression should not lead investigators to over-individualize the issue of job-related distress. Depression's etiology is best understood through the relationship between internal dispositions and external conditions $[3,8]$. Therefore, neglecting the role of working conditions (e.g., excessive workloads, contradictory work goals) in the development of occupational depression would be misguided. A coordinated examination of individual and organizational factors is needed.

The SARS-CoV-2 outbreak has challenged the resilience of medical professionals. Such circumstances render the need for valid and reliable assessments of job-related distress particularly salient. Record-high suicide rates among police officers or rising attrition rates among schoolteachers highlight this need as well. The issue of occupational health is too serious to be addressed with loose indicators and shaky constructs. We believe that a change in paradigm from burnout to occupational depression can make a difference in our ability to protect the health of our workforce.

Author Contributions. Conceptualization: R.B., I.S.S.; Formal analysis: R.B., I.S.S.; Investigation: R.B., I.S.S.; Writing-original draft: R.B.; Writing-review and editing: R.B., I.S.S.

Conflicts of Interest. The authors declare none.

\section{References}

1. Rotenstein LS, Torre M, Ramos MA, Rosales RC, Guille C, Sen S, et al. Prevalence of burnout among physicians: a systematic review. JAMA. 2018; 320(11):1131-50. doi:10.1001/jama.2018.12777.

2. Schwenk TL, Gold KJ. Physician burnout-a serious symptom, but of what? JAMA. 2018;320(11):1109-10. doi:10.1001/jama.2018.11703.

3. Bianchi R, Verkuilen J, Schonfeld IS, Hakanen JJ, Jansson-Fröjmark M, Manzano-García G, et al. Is burnout a depressive condition? A 14-sample meta-analytic and bifactor analytic study. Clin Psychol Sci. 2021;9(4): 579-97. doi:10.1177/2167702620979597.

4. Maslach C, Jackson SE, Leiter MP. Maslach Burnout Inventory manual. 4th ed. Palo Alto, CA: Consulting Psychologists Press; 2016.

5. Guseva Canu I, Marca SC, Dell'Oro F, Balázs Á, Bergamaschi E, Besse C, et al. Harmonized definition of occupational burnout: a systematic review, semantic analysis, and Delphi consensus in 29 countries. Scand J Work Environ Health. 2021;47(2):95-107. doi:10.5271/sjweh.3935.

6. Sterkens P, Baert S, Rooman C, Derous E. Why making promotion after a burnout is like boiling the ocean (IZA DP No. 14502). Bonn: Institute of Labor Economics (IZA); 2021. https://ftp.iza.org/dp14502.pdf October 1, 2021.

7. Heinemann LV, Heinemann T. Burnout research: emergence and scientific investigation of a contested diagnosis. SAGE Open. 2017;7(1): 2158244017697154. doi:10.1177/2158244017697154.

8. Bianchi R, Schonfeld IS. The Occupational Depression Inventory: a new tool for clinicians and epidemiologists. J Psychosom Res. 2020;138:110249. doi:10.1016/j.jpsychores.2020.110249.

9. Meier ST, Kim S. Meta-regression analyses of relationships between burnout and depression with sampling and measurement methodological moderators. J Occup Health Psychol. 2021. doi:10.1037/ocp0000273.

10. Bianchi R, Schonfeld IS. The Occupational Depression Inventory-a solution for estimating the prevalence of job-related distress. Psychiatry Res. 2021;305:114181. doi:10.1016/j.psychres.2021.114181. 